\title{
3D Numerical Simulation of Kuroshio-induced Wake Near Green Island, Taiwan
}

\author{
Tien-Hung Hou \\ epartment of Marine Environmental Engineering, National Kaohsiung University of Science and Technology, \\ Kaohsiung City, Taiwan \\ Jen-Yi Chang \\ Tainan University of Technology, Tainan City, Taiwan \\ Chia-Cheng Tsai \\ Department of Marine Environmental Engineering, National Kaohsiung University of Science and Technology, \\ Kaohsiung City, Taiwan, tsaichiacheng@nkust.edu.tw
}

Tai-Wen Hsu

Center of Excellence for Ocean Engineering, National Taiwan Ocean Univer-sity, Keelung, Taiwan, twhsu@mail.ntou.edu.tw

Follow this and additional works at: https://jmstt.ntou.edu.tw/journal

Part of the Fresh Water Studies Commons, Marine Biology Commons, Ocean Engineering Commons, Oceanography Commons, and the Other Oceanography and Atmospheric Sciences and Meteorology Commons

\section{Recommended Citation}

Hou, Tien-Hung; Chang, Jen-Yi; Tsai, Chia-Cheng; and Hsu, Tai-Wen (2021) "3D Numerical Simulation of Kuroshioinduced Wake Near Green Island, Taiwan," Journal of Marine Science and Technology. Vol. 29: Iss. 3, Article 7. DOI: $10.51400 / 2709-6998.1436$

Available at: https://jmstt.ntou.edu.tw/journal/vol29/iss3/7

This Research Article is brought to you for free and open access by Journal of Marine Science and Technology. It has been accepted for inclusion in Journal of Marine Science and Technology by an authorized editor of Journal of Marine Science and Technology. 
3D Numerical Simulation of Kuroshio-induced Wake Near Green Island, Taiwan Acknowledgements

No more

This research article is available in Journal of Marine Science and Technology: https://jmstt.ntou.edu.tw/journal/ 


\title{
3D Numerical Simulation of Kuroshio-induced Wake Near Green Island, Taiwan
}

\author{
Tien-Hung Hou ${ }^{a}$, Jen-Yi Chang ${ }^{b}$, Chia-Cheng Tsai ${ }^{a, c, d, *}$, Tai-Wen Hsu ${ }^{d, * *}$ \\ ${ }^{a}$ Department of Marine Environmental Engineering, National Kaohsiung University of Science and Technology, Kaohsiung City, \\ Taiwan \\ b Tainan University of Technology, Tainan City, Taiwan \\ ${ }^{c}$ Department of Marine Environment and Engineering, National Sun Yat-sen University, Kaohsiung City, Taiwan \\ ${ }^{\mathrm{d}}$ Center of Excellence for Ocean Engineering, National Taiwan Ocean University, Keelung, Taiwan
}

\begin{abstract}
In this research, Kuroshio-induced vortex shedding in the lee of Green Island was numerically studied using the threedimensional Princeton Ocean Model. The temporal and spatial characteristics of the vortex street were analyzed at different tidal levels, wind fields and water depths. The numerical results were validated by comparing with in-situ measurements from the Green Island tidal station, shipboard acoustic doppler current profiler (ADCP), conductivitytemperature-depth (CTD) profiler, and moderate resolution imaging spectroradiometer (MODIS) satellite image datasets. Also, the three-dimensional results of velocity, temperature, and salinity fields were compared well with observational data. Furthermore, the influence depths of the wind effect and vortex shedding were found to be approximately 100 and $300 \mathrm{~m}$, respectively. The aspect ratio and dimensionless width were evaluated to be 3.64 and 1.46, respectively. In addition, the temporal scales (Strouhal numbers), and propagation speeds were analyzed at various water depths. Finally, the elongation of the Kuroshio-induced vortex street was studied under wind and tide effects at various water depths.
\end{abstract}

Keywords: Ocean modeling, Green Island wake, 3D realistic model, Wind stress

\section{Introduction}

$\mathrm{T}$ aiwan has extremely limited domestic energy resources and must rely on oil and coal imports to satisfy the majority of its energy demand [1]. The Kuroshio Current is known for its strong and fast flows all year around and could be a potential source of energy [2]. Chen [3] predicted that the energy of the Kuroshio Current in the eastern coastal waters of Taiwan is approximately $30 \mathrm{GW}$ and proposed several conceptual designs for Kuroshio power plants. Currently, the Taiwan Power Company provides 34 GW of power from all types of energy resources [4]. If energy of the Kuroshio Current can be explored and effectively used, Taiwan can reduce its dependence on the imported energy.

The Kuroshio Current originates from the North Equatorial Current and flows northward parallel to the eastern shoreline of Taiwan. The Kuroshio Current has a width of approximately $100-150 \mathrm{~km}$, a speed ranging $1.0-1.5 \mathrm{~m} \mathrm{~s}^{-1}$, and is motionless at a depth of over $400 \mathrm{~m} \mathrm{[5-7].} \mathrm{The} \mathrm{volume} \mathrm{of} \mathrm{transport}$ of the Kuroshio Current varies between 10.46 and $22.92 \mathrm{~Sv}$ [8].

In order to locate a site for Kuroshio power plants, several factors should be considered: (1) vicinity to coast, (2) shallow seabed, (3) stable flow velocity,

Received 31 October 2019; revised 19 February 2020; accepted 8 September 2020.

Available online 25 June 2021.

* Corresponding author. Department of Marine Environmental Engineering, National Kaohsiung University of Science and Technology, Kaohsiung City, Taiwan.

** Corresponding author.

E-mail addresses: tsaichiacheng@nkust.edu.tw (C.-C. Tsai), twhsu@mail.ntou.edu.tw (T.-W. Hsu).

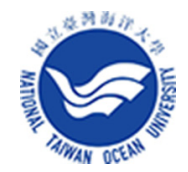


and (4) high flow speed [9]. Green Island is located at $121^{\circ} 29^{\prime} \mathrm{E}, 22^{\circ} 39^{\prime} \mathrm{N}$ and is $40 \mathrm{~km}$ off the southeastern coast of Taiwan with the passage of energetic Kuroshio as shown in Fig. 1. Therefore, Green Island is usually considered a site for the extraction of energy from the Kuroshio Current. Usually, the plant site is selected on the lee side of the Green Island to take off the Kuroshio Current $[4,10]$.

It is well known that when a flow passing a cylindrical body, it generates a so-called "von Kármán vortex sheet" downstream [11]. As previous studies have indicated [12-14], the types of wake and vortex shedding are dictated by the Reynolds number $R e=$ $u_{\infty} L / v$, where $u_{\infty}, L$, and $v$ are the representative velocity, diameter of the cylinder, and kinematic eddy viscosity, respectively. When $\operatorname{Re}<<1$, the flow field is laminar and symmetric. When $4<\operatorname{Re}<40$, the symmetry disappears, a laminar separation occurs, and two attached eddies are formed behind the obstacle. A periodic von Kármán vortex street can be observed as $40<\operatorname{Re}<1000$, and the separated flow becomes turbulent when $R e>1000$. Based on field observations downstream of Green Island [15], the evaluated Reynolds number is approximately 91. Therefore, it is desirable to study the Kuroshioinduced wake in the lee of Green Island when a site of Kuroshio power plants is selected to be located there.

The Kuroshio-induced wake in the lee of Green Island has been observed using the shipboard acoustic doppler current profiler (ADCP), conductivity-temperature-depth (CTD) profiler [15-17], and moderate resolution imaging spectroradiometer (MODIS) satellite image dataset [18-21]. These data can be used to analyze the physical phenomena of the vortex shedding or to develop numerical models.

Numerical models can be used to obtain the flow fields of the Kuroshio-induced wake in the lee of Green Island for a spatial domain during a certain time period. Such numerical results can complement the field measurements when studying the physical phenomena of the wake. Usually, a numerical ocean model is driven by certain forcing conditions of the boundary velocity, temperature, salinity, tide and/or wind in two or three dimensions. Most of the previous researches are scenario studies driven by some artificial forcing conditions in two [13,21,22] or three [23] dimensions.

When numerical models are used to realistically simulate the Kuroshio-induced wakes, two-dimensional results can be found in the previous studies [4,12]. For example Hsu, et al. [12]; modelled the typhoon effect on the Kuroshio-induced wake, and $\mathrm{Hsu}$, et al.[4] compared the results from two- and three-dimensional ocean models. They indicated that the improved accuracy by a three-dimensional model over a two-dimensional model is limited. Therefore, only two-dimensional flow fields were presented in their study. A comprehensive

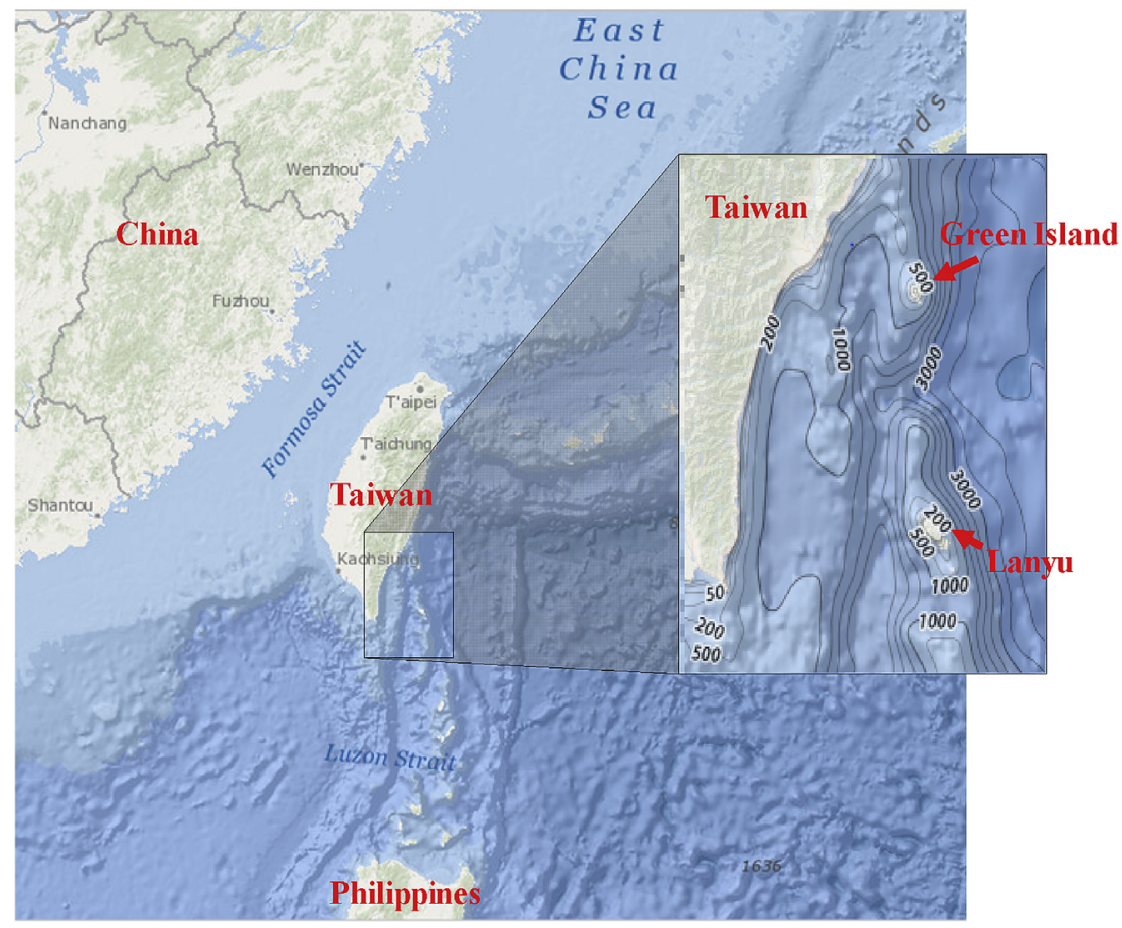

Fig. 1. Location of Green island. (Source: NCEI). 
Table 1. Numerical model conditions by boundary force in previous studies.

\begin{tabular}{llllll}
\hline Authors (Year) & Dim. & \multicolumn{4}{l}{ Forcing conditions } \\
\cline { 3 - 6 } & & T/S & U/V & Tide & Wind \\
\hline Huang, et al. (2014) [21] & 2D & $\mathrm{X}$ & $\mathrm{S}$ & $\mathrm{X}$ & $\mathrm{X}$ \\
Hsu, et al. (2015a) [12] & 2D & $\mathrm{X}$ & $\mathrm{S}$ & $\mathrm{X}$ & $\mathrm{R}$ \\
Hsu, et al. (2015b) [13] & 2D & $\mathrm{X}$ & $\mathrm{S}$ & $\mathrm{X}$ & $\mathrm{S}$ \\
Hsu, et al. (2015c) [4] & 2D & $\mathrm{R}$ & $\mathrm{R}$ & $\mathrm{R}$ & $\mathrm{R}$ \\
Liu and Chang (2018) [23] & 3D & $\mathrm{S}$ & $\mathrm{S}$ & $\mathrm{X}$ & $\mathrm{X}$ \\
Present study & 3D & $\mathrm{R}$ & $\mathrm{R}$ & $\mathrm{R}$ & $\mathrm{R}$ \\
\hline
\end{tabular}

$\mathrm{R}$ : realistic simulation; S: scenario study; $\mathrm{X}$ : not considered.

comparison of these studies are addressed in Table 1. In the present study, the three-dimensional Princeton Ocean Model (3D POM) is adopted to realistically simulate the flow field of the Kuroshioinduced wake in the lee of Green Island. Furthermore, different wind velocities, temperature and salinity conditions are incorporated in the model as the driving forces to explore changes in the Green Island wakes.

The 3D POM was developed in the late 1970s by Blumberg and Mellor, with subsequent contributions from other researchers. It is formulated in the primitive equations with a second-moment turbulence closure sub-model $[24,25]$ to provide vertical mixing coefficients. In addition, a curvilinear sigma coordinate system and orthogonal Cartesian coordinates, with an "Arakawa $\mathrm{C}^{\text {" differencing }}$ scheme, are used in the vertical and horizontal directions, respectively. Furthermore, the nested boundary condition [26] is used in the present study to improve the mesh resolution and reduce the execution time.

Overall, the 3D POM is used to realistically simulate the flow fields of the Kuroshio-induced wake in the lee of the Green Island. The model is driven by the boundary velocity, temperature, salinity, tide and wind. To validate the numerical model, the results are compared with in-situ measurements from the shipboard ADCP, CTD profiler, and MODIS satellite image datasets. Furthermore, numerical results are adopted to analyze the characteristics of vortex shedding, including the spatial lengths (aspect ratio and dimensionless width), temporal scale (Strouhal number) and propagation speed. The physical phenomena of the Kuroshioinduced wake in the lee of Green Island are discussed herein.

\section{Numerical model}

In the 3D POM, the governing equations [27] are described by the conservations of mass and momentum under assumptions of the incompressibility, the rigid-lid free surface condition, the turbulence closure condition, and the Boussinesq approximation, which can be presented in Cartesian coordinates as follows:

$$
\begin{aligned}
& \frac{\partial U}{\partial x}+\frac{\partial V}{\partial y}+\frac{\partial W}{\partial z}=0 \\
& \frac{\partial U}{\partial t}+U \frac{\partial U}{\partial x}+V \frac{\partial U}{\partial y}+W \frac{\partial U}{\partial z}-f V \\
& \quad=-\frac{1}{\rho_{0}} \frac{\partial P}{\partial x}+\frac{\partial}{\partial z}\left(K_{M} \frac{\partial U}{\partial z}\right)+F_{u}
\end{aligned}
$$

$\frac{\partial V}{\partial t}+U \frac{\partial V}{\partial x}+V \frac{\partial V}{\partial y}+W \frac{\partial V}{\partial z}+f U=-\frac{1}{\rho_{0}} \frac{\partial P}{\partial y}+\frac{\partial}{\partial z}\left(K_{M} \frac{\partial V}{\partial z}\right)+F_{v}$

$0=-\frac{\partial P}{\partial z}+\rho g$

$\frac{\partial T}{\partial t}+U \frac{\partial T}{\partial x}+V \frac{\partial T}{\partial y}+W \frac{\partial T}{\partial z}=\frac{\partial}{\partial z}\left(K_{H} \frac{\partial T}{\partial z}\right)+F_{T}$

$\frac{\partial S}{\partial t}+U \frac{\partial S}{\partial x}+V \frac{\partial S}{\partial y}+W \frac{\partial S}{\partial z}=\frac{\partial}{\partial z}\left(K_{H} \frac{\partial S}{\partial z}\right)+F_{S}$

where $U, V, W, T, S$, and $P$ are the mean velocity components, temperature, salinity and hydrostatic pressure. And, densities are defined by $\rho=\rho_{0}+\rho^{\prime}$ with $\rho, \rho_{0}$, and $\rho^{\prime}$ being the total density of the seawater, the reference density of the seawater and the perturbation density, respectively. In addition, $K_{M}$ is the vertical eddy viscosity diffusivity of turbulent momentum mixing and $K_{H}$ is a vertical eddy viscosity diffusivity of the turbulence of heat and salt. Furthermore, $g$ and $f$ represent the gravity acceleration and the Coriolis force, respectively. Finally, $F_{u}, F_{v}, F_{T}$, and $F_{S}$ are diffusion coefficients which are usually required to damp small scale numerical modes.

In the horizontal directions, the computation is performed in two nested domains, as summarized in Table 2. The global domain covers the regions of ocean near Taiwan (TW), between $117.0^{\circ} \mathrm{E}-125.0^{\circ} \mathrm{E}$ and $18.0^{\circ} \mathrm{N}-27.0^{\circ} \mathrm{N}$ (Fig. 2, left), the horizontal resolution is $1 / 16^{\circ}$ (approximately $6.25 \mathrm{~km}$ ). The other domain is a local domain that covers the Taitung

Table 2. The domain of these models.

\begin{tabular}{llll}
\hline Model & Range & Grid sizes & Resolution (degree) \\
\hline TW & $117.0^{\circ} \mathrm{E}-125.0^{\circ} \mathrm{E}$ & $129 \times 145 \times 31$ & $1 / 16(0.045)$ \\
& $18.0^{\circ} \mathrm{N}-27.0^{\circ} \mathrm{N}$ & & \\
TOO & $120.88^{\circ} \mathrm{E}-122.5^{\circ} \mathrm{E}$ & $163 \times 220 \times 31$ & $1 / 100(0.01)$ \\
& $22.06^{\circ} \mathrm{N}-24.25^{\circ} \mathrm{N}$ & & \\
\hline
\end{tabular}




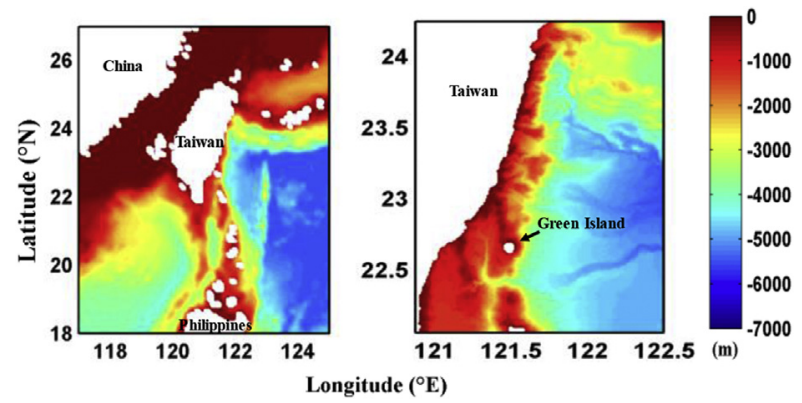

Fig. 2. Bathymetry in the nested model domains of 3D POM model: (left) Taiwan near the ocean; (right) Taitung Open Ocean.

Open Ocean (TOO), between $120.88^{\circ} \mathrm{E}-122.5^{\circ} \mathrm{E}$ and $22.06^{\circ} \mathrm{N}-24.25^{\circ} \mathrm{N}$ (Fig. 2, right), whose horizontal resolution is $1 / 100^{\circ}$ (approximately $1.0 \mathrm{~km}$ ). In addition, in the vertical direction, there are 31 sigma levels, and the depth of the water ranges $10-5500 \mathrm{~m}$. These bathymetry data are based on the 1 arc-minute resolution global surface (ETOPO1) provided by the National Geographical Data Center, NOAA [28], and $200 \mathrm{~m}$ resolution of the Taiwan Digital Bathymetry Model, Version 6 (TaiDBMv6) provided by the Ocean Data Bank [29].

The 3D POM uses the mode-splitting scheme, which integrate the barotropic (external) and baroclinic (internal) modes. These modes are coupled at the source-code level. Here, the external mode uses a shorter time step, DTE, whereas the internal model utilizes a longer time step, DTI. The external mode primarily provides the surface elevation to the internal mode, and the internal mode provides depth-averaged integrals of momentum advection, density and bottom stress to the external mode. In this study, the configurations of global and local domains are as shown in Table 3.

As summarized in Table 3, the forcing conditions of the tide and wind are specified for the TOO model. Here, the wind data are obtained from the regional spectral model (RSM) [30]. The RSM was developed at the National Center for Environmental Predictions with spatial and time resolutions of $0.25^{\circ}$ and $3 \mathrm{~h}$, respectively. In addition, the TOO forcing condition of the boundary tide is set with the tidal elevation and barotropic velocity derived from OSU TOPEX/Poseidon Global Inverse Solution TPXO

Table 3. Configurations of these models.

\begin{tabular}{llllll}
\hline Model & DTE & DTI & Forcing conditions & \\
\cline { 4 - 6 } & & & Ocean Datasets & Tide & Wind \\
\hline TW & 5.0 & 150.0 & HYCOM & X & X \\
TOO & 1.0 & 30.0 & TW & O & O \\
\hline
\end{tabular}

[31,32]. The eleven major constituents (M2, S2, N2, $\mathrm{K} 2, \mathrm{~K} 1, \mathrm{O} 1, \mathrm{P} 1, \mathrm{Q} 1, \mathrm{M} 4, \mathrm{Mm}$ and $\mathrm{Mf}$ ) are included. Furthermore, the initial and boundary conditions of the TW model are set form the Global Hybrid Coordinate Ocean Model (HYCOM) [33] and its spatial and time resolution are $1 / 12^{\circ}$ and 1 day, respectively.

In both the TW and TOO models, the boundary conditions of the external mode are the given elevation and velocity radiation conditions, which are defined in (A-2) and (A-4) in the POM user guide [34], respectively. Furthermore, the boundary conditions of the internal mode are the velocity radiation and temperature/salinity upstream advection conditions, which are formulated in (B-2) and (B-3) in the POM user guide, respectively. When applying these boundary conditions, the ocean datasets from the lower-resolution models are used as depicted in Fig. 3. In addition, the nudging scheme $[35,36]$ is utilized to smooth these input data in both time and space.

\section{Model validation}

In order to validate the proposed model, a simulation was performed from September 1 to September 30, 2010, UTC according to the previous study [15]. The numerical results are compared with the surface elevation from the tidal station and the observed two- and three-dimensional velocity fields from the shipboard ADCP, CTD profiler, and MODIS satellite image datasets. In addition, the present results are also compared with the lowresolution results from HYCOM to indicate the improvement of the proposed model with discussions on the effects of the tide and wind.

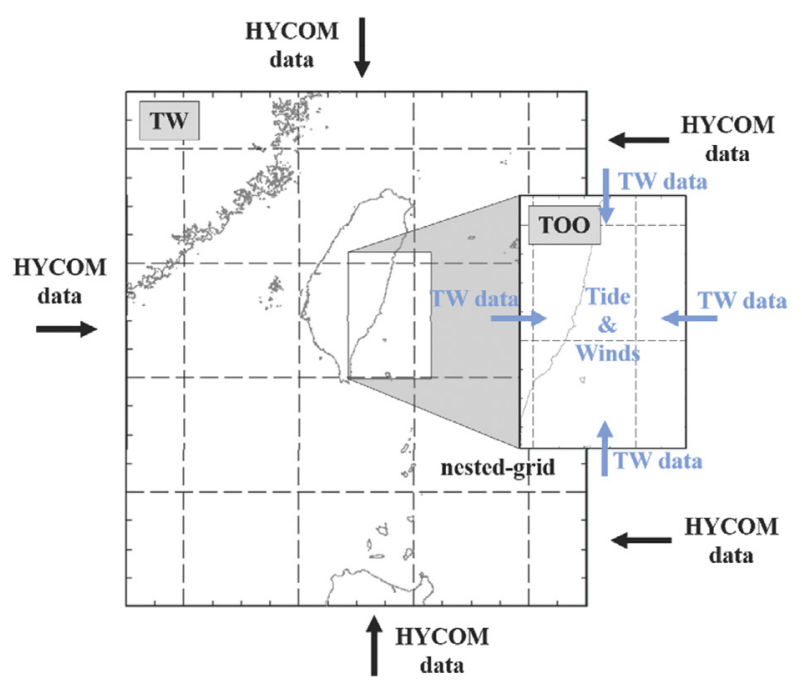

Fig. 3. Sketch of boundary conditions of TW and TOO models. 


\subsection{Surface elevation compared with measurements from Green Island tidal station}

First, the surface elevation obtained by the present model is compared with the measurements from the Green Island tidal station, which is located at latitude $22.65^{\circ} \mathrm{N}$ and longitude $121.45^{\circ} \mathrm{E}$. The time histories of the tidal elevations are depicted in Fig. 4. Sequentially, the root-meansquare error between the numerical results and the measurements can be evaluated to be 0.0391 , which shows an excellent agreement. This result indicate that the present model can simulate the tidal motion well.

The corresponding surface velocity fields of the TW and TOO models are depicted in Fig. 5. In the figure, a Kuroshio-induce vortex shedding can be observed. More details will be discussed below.

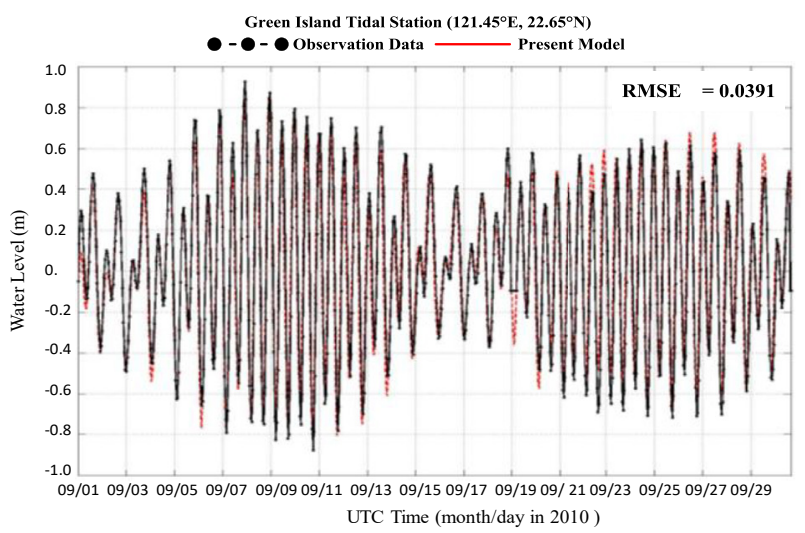

(a) $2010 / 09 / 01-2010 / 09 / 30$

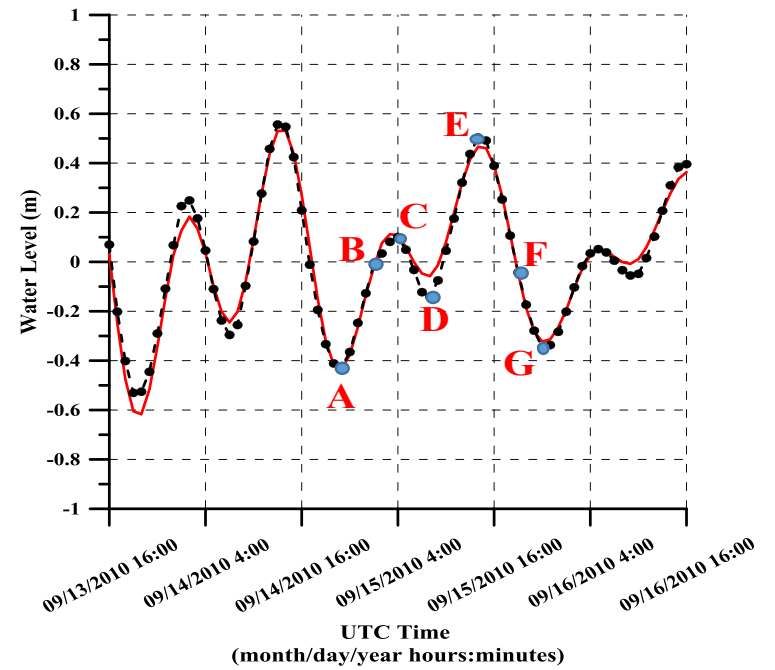

(b) $2010 / 09 / 13$ 16:00-2010/09/16 16:00

Fig. 4. Time history of water surface elevation in (a) 1 month and (b) 3 days.

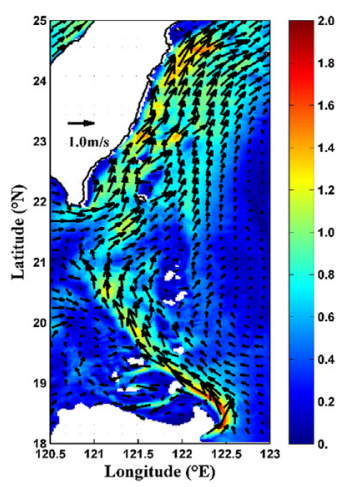

(a)

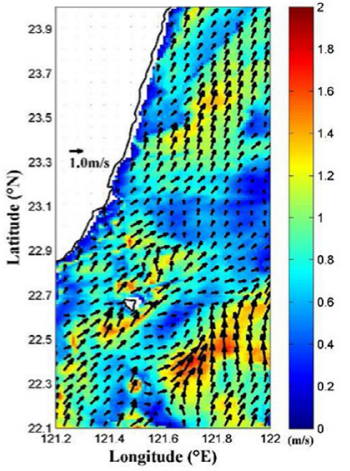

(b)
Fig. 5. Numerical simulations of Kuroshio-induced wake in the lee of Green Island using(a) TW and (b) TOO models.

\section{2. $2 D$ Comparison with the datasets from MODIS satellite image and shipboard $A D C P$}

The surface velocity fields and corresponding streamlines obtained by the present model are then compared with the datasets from the shipboard ADCP and MODIS satellite images, respectively. Fig. 6 shows the MODIS satellite image [15] and the corresponding surface streamlines at 4:55 a.m. September 15, 2010, GMT. In the figure, similar vortex shedding characteristics can be observed.

Sequentially, a comparison is made for the detailed surface velocity fields obtained by the Shipboard ADCP measurements [15] and the present model as depicted in Fig. 7. A similar result can also be observed while the present numerical results seem to be slightly overestimated, which require further investigations. Therefore, the present results indicate that the proposed model can simulate well the surface velocity field of the Kuroshio-induce vortex shedding in the lee of Green Island.

\subsection{Comparison of three-dimensional results}

When numerical models are used to realistically simulate the Kuroshio-induced wakes, most of the

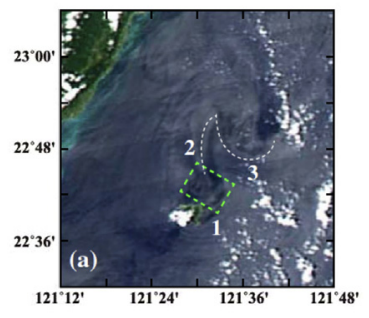

(a) Satellite imagery

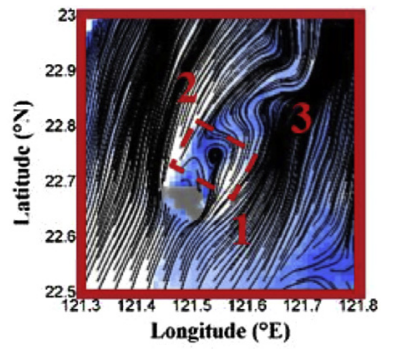

(b) Present model
Fig. 6. Characteristics of vortex shedding at 4:55 A.M. 15 September, 2010 GMT obtained by (a) MODIS satellite image and (b) the present model. 


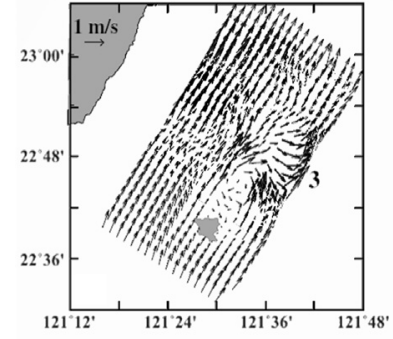

(a) Shipboard ADCP measurement

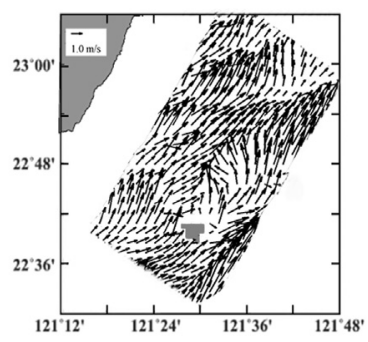

(b) Present model
Fig. 7. Surface velocity field at 4:55 a.m. September 15, 2010, GMT obtained from (a) the shipboard ADCP measurement and (b) the present model. previous studies have focused on two-dimensional velocity fields $[4,12]$. Here, the three-dimensional velocity fields obtained from the shipboard ADCP [15] are compared with those obtained from the present model at 4:55 a.m. September 15, 2010, GMT. Fig. 8 shows the measured velocity field and numerical results along transects L3 and L5. In the figure, similar results can be observed. In addition, a strong velocity shear is present behind Green Island. This strong velocity shear causes severe instability and mixing.

In addition, comparisons are made for the threedimensional temperature and salinity fields obtained from the CTD profiler [15] and the present

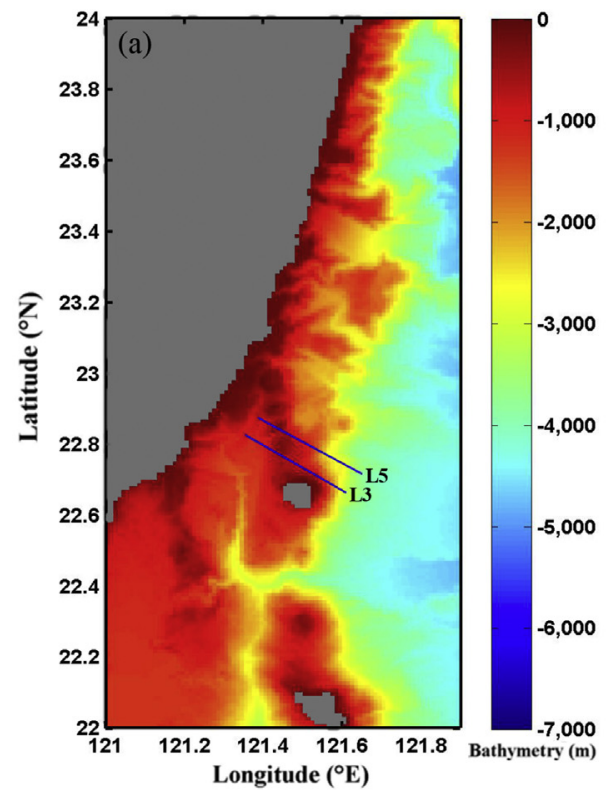

(b)

Measurement
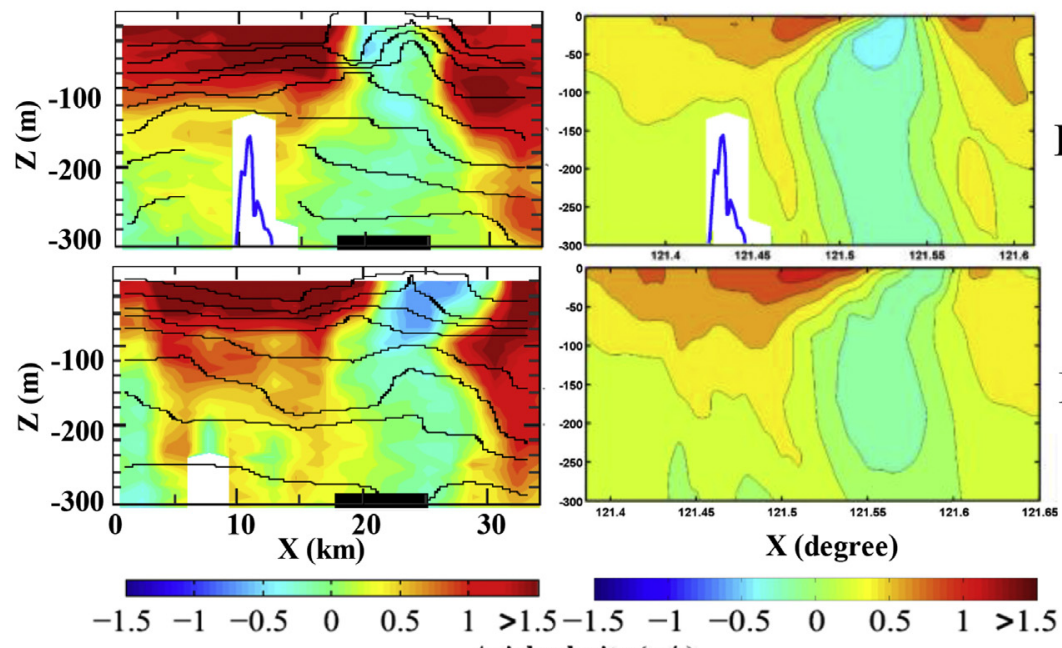

L3

L5

Fig. 8. (a) Survey routes L3 and L5 and (b) the cross-wake northeastward views of velocity fields from the shipboard ADCP measurement and the present model. 
(a)

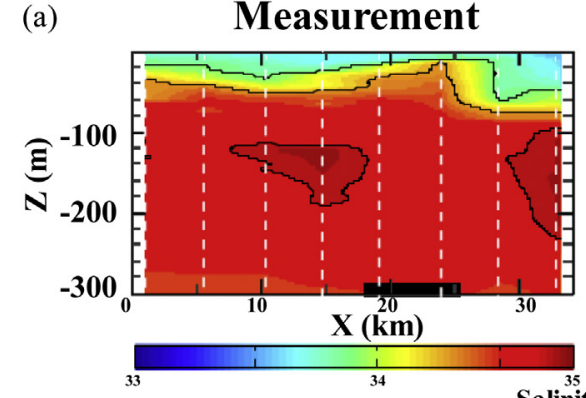

(b)

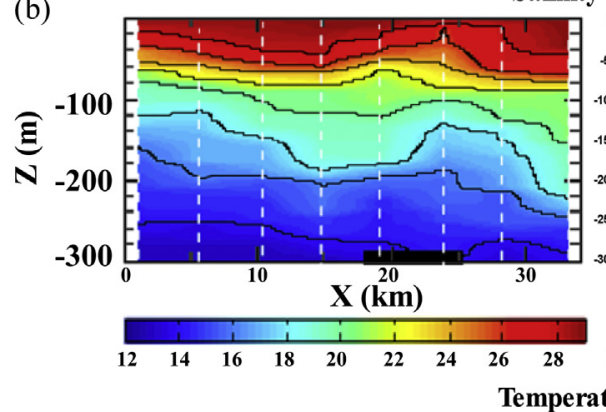

Temperature $\left({ }^{\circ} \mathrm{C}\right)$
Present model

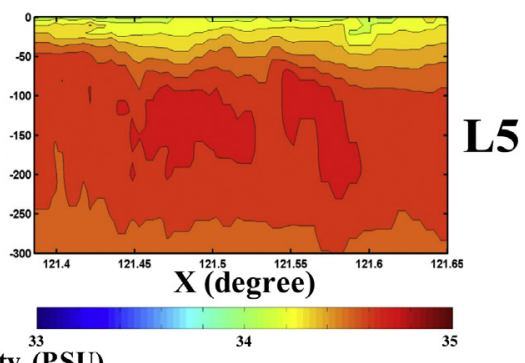

L5

L5

Fig. 9. Cross-wake northeastward views of (a) temperature and (b) salinity fields from the CTD profiler and the present model.

model at 4:55 a.m. September 15, 2010, GMT. Fig. 9 shows the temperature and salinity fields from the CTD and numerical model along transect L5. In the figure, a good agreement can also be observed.

Overall, the comparisons (Figs. 4-9) show that the proposed model can realistically simulate the surface elevation as well as the two- and threedimensional velocity, temperature, and salinity fields of the Kuroshio-induce vortex shedding around Green Island.

\subsection{Comparison with HYCOM data}

Although the model is validated, the effects on various realistic forcing conditions of boundary current, tide and wind have to be studied. Because the TW model uses the results from the HYCOM model as the input data, it is interesting to compare the results from the HYCOM and present models. The effects of the tide and wind are not considered in the HYCOM results. Therefore, we also conducted several scenario studies using the present model based on various settings of forcing conditions, as shown in Fig. 10. As shown in the figure, the results (Fig. 10a and b) of the HYCOM and the present model with solely the boundary-current input are similar whereas the latter is of higher resolution. It can also be observed that the effects of the tide and wind are also significant in Fig. 10. To be more specific, the results obtained by the present model, with all the forcing conditions of the boundary current, tide, and wind, reveal an obvious vortex shedding as depicted in Fig. 10d. However, the vortex shedding is considerably less observable when only the forcing condition of the boundary current is considered as shown in Fig. 10(a) and (b). A comparison on the wind effect will be stated later based on Fig. 10(c) and (d).

These results imply that the effects of the realistic forcing conditions on the boundary current, tide, and wind are significant and that their uses are relevant and necessary when the present model is applied to realistically simulate the Kuroshioinduced vortex shedding around Green Island.

\section{Results and discussion}

After the model is validated, scenario studies will be performed to investigate the influence depths of the wind effect and the vortex shedding. Then, analysis will be conducted to study the characteristics of vortex shedding, including the spatial lengths (aspect ratio and dimensionless width), temporal scales (Strouhal number) and propagation speeds.

\subsection{Three-dimensional effects}

The Kuroshio-induce vortex shedding is a very complicated phenomenon, which is affected by several different factors including the wind, tide, bathymetry, mesoscale flows, Coriolis force, coastline changes, and others. It is difficult to find an easy way to analyze and quantify the three-dimensional 


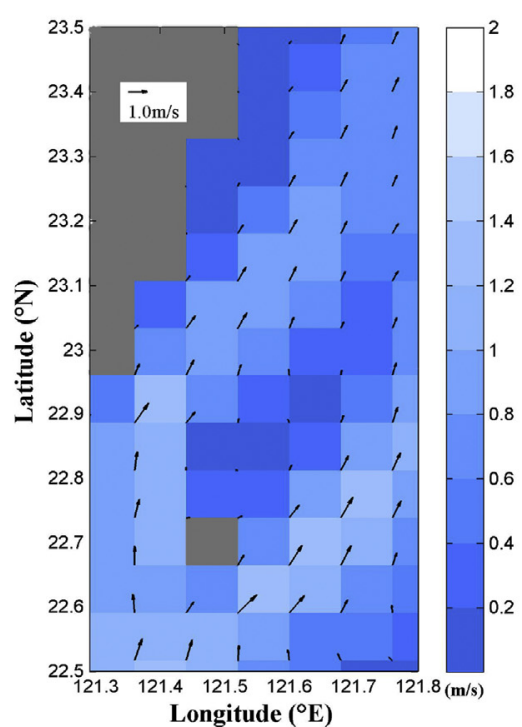

(a) HYCOM [C]

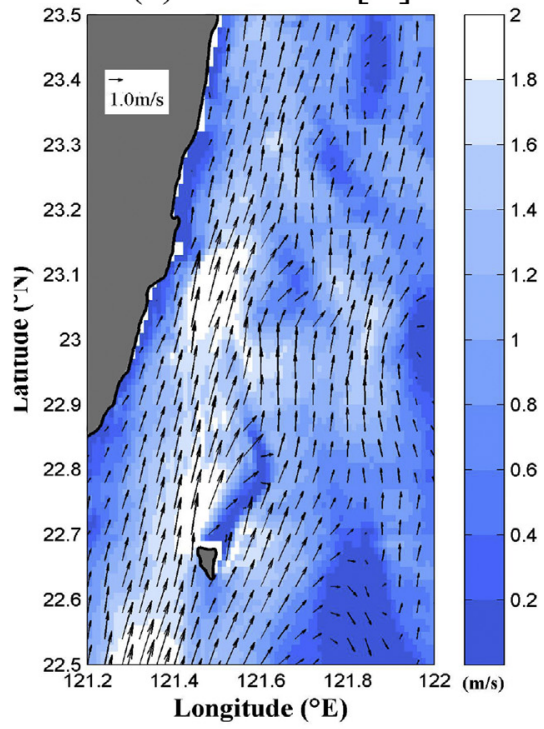

(c) Model $[\mathrm{C}+\mathrm{T}]$

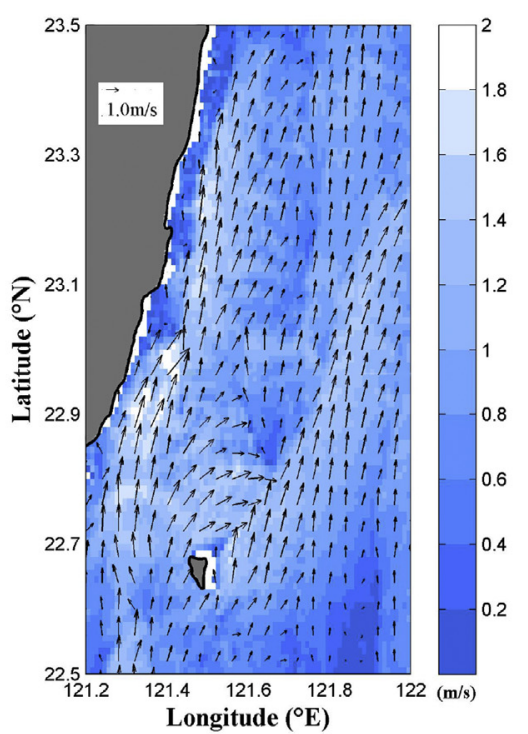

(b) Model [C]

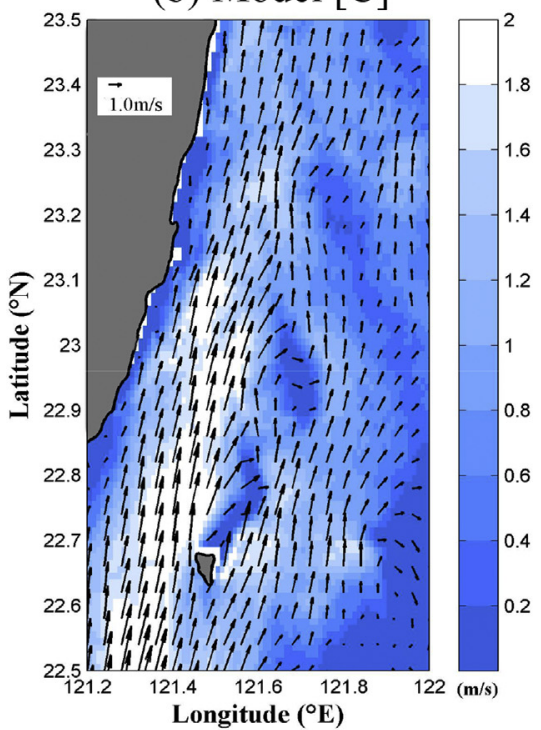

(d) Model $[\mathrm{C}+\mathrm{T}+\mathrm{W}]$

Fig. 10. Surface velocity fields at 00:00 UTC on 09/15/2010 obtained by (a) HYCOM models, and (b)-(d) the present model with various forcing conditions. (C, boundary current; $T$, tide; $W$, wind).

influences of these factors. Here, a simple method is utilized to investigate the influence depth of the wind effect.

The realistic simulation is compared with the corresponding scenario study, which has the same forcing conditions but no wind input. The surface velocity fields of these two simulations are given in Fig. 10(d) and (c), respectively. Correspondingly, we have the velocity fields at the water depths of 50 , 100, and $150 \mathrm{~m}$, as depicted in Fig. 11. In the figure, it is clear that the wind can affect the flow fields to approximately $100 \mathrm{~m}$ deep whereas the vortex shedding occurs deeper than $150 \mathrm{~m}$. In other words, Figs. 10(d), 11(a), and 11(b) are significantly different from the corresponding no-wind results in Figs. $10(\mathrm{c}), 11(\mathrm{~d})$, and 11(e), respectively.

Then, the $U$ velocity contours are collected to form a three-dimensional plot in Fig. 12. Here, the $U$ velocity contours are used to easily visualize the vortex shedding as the inflow is mainly in the V direction. From Fig. 12, it is clear that the influence depth of the vortex shedding is deeper than $300 \mathrm{~m}$.

To analyze the three-dimensional influences of different forcing factors, more thorough studies with careful and complete scenario designs are required. These are beyond the scope of this study, however, and are currently under investigation. 

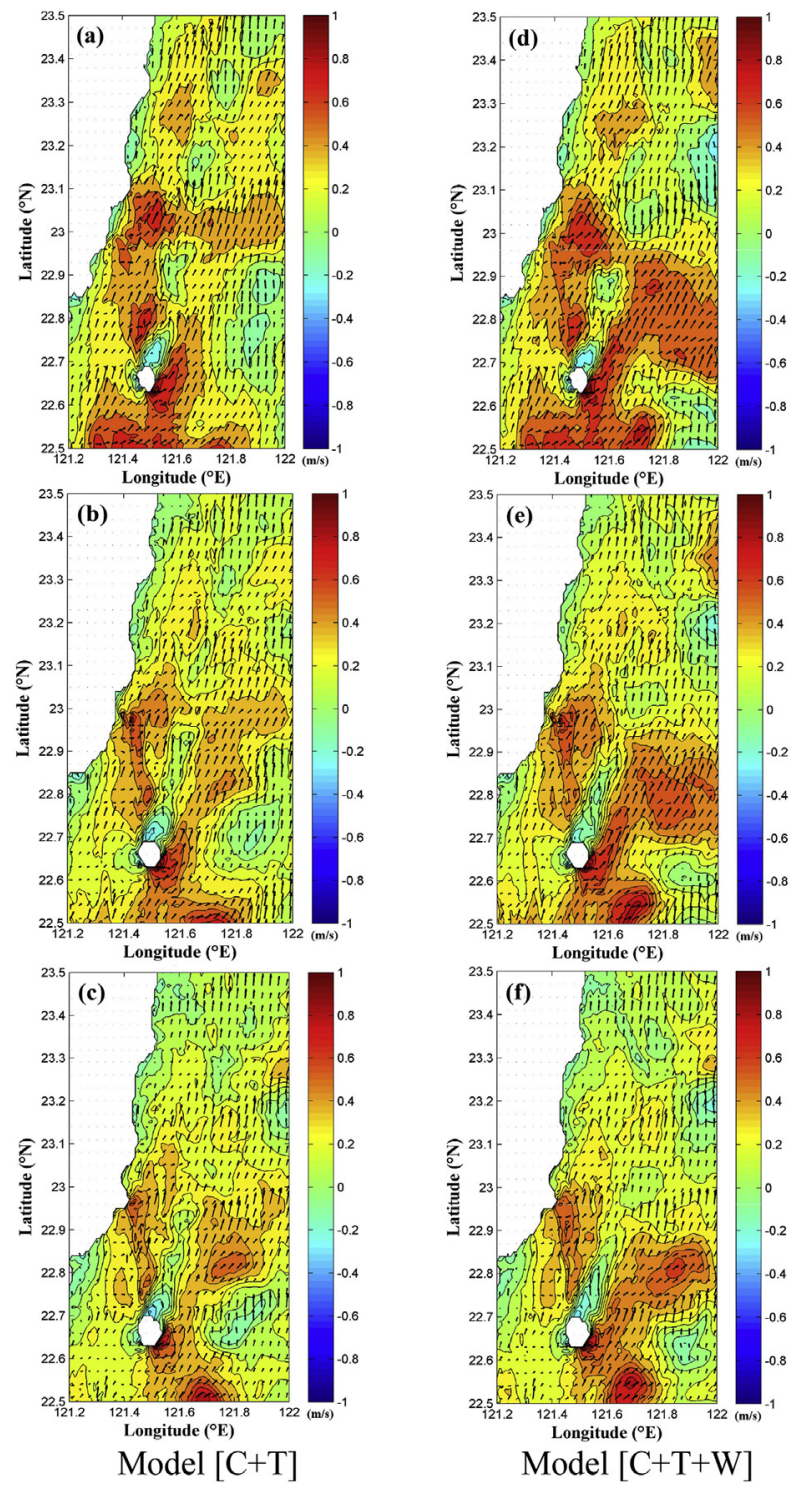

Fig. 11. Velocity field and $U$ velocity contours at water depths of $(a),(d)$ $50 \mathrm{~m}$, (b), (e) $100 \mathrm{~m}$, and (c), (f) $150 \mathrm{~m}$ for the simulations with and without winds (00:00 UTC on 09/15/2010).

\subsection{Characteristics of vortex shedding}

The characteristics of vortex shedding can be described by several dimensionless quantities. As depicted in Fig. 13, definitions are given for the streamwise length $(a)$, transverse length $(b)$, and diameter $(L)$ (for example, the diameter of Green island, $L=5 \mathrm{~km}$ ). Following [4], the aspect ratio and dimensionless width are defined as $A r=a / b$ and $B r=b / L$, respectively. A typical result is given in Fig. 13b. In addition, the vortex shedding can be characterized by the Strouhal number $(S t)$ and relative propagation speed $(U p)$ as follows:

$S t=L /\left(u_{\infty} T\right)$,

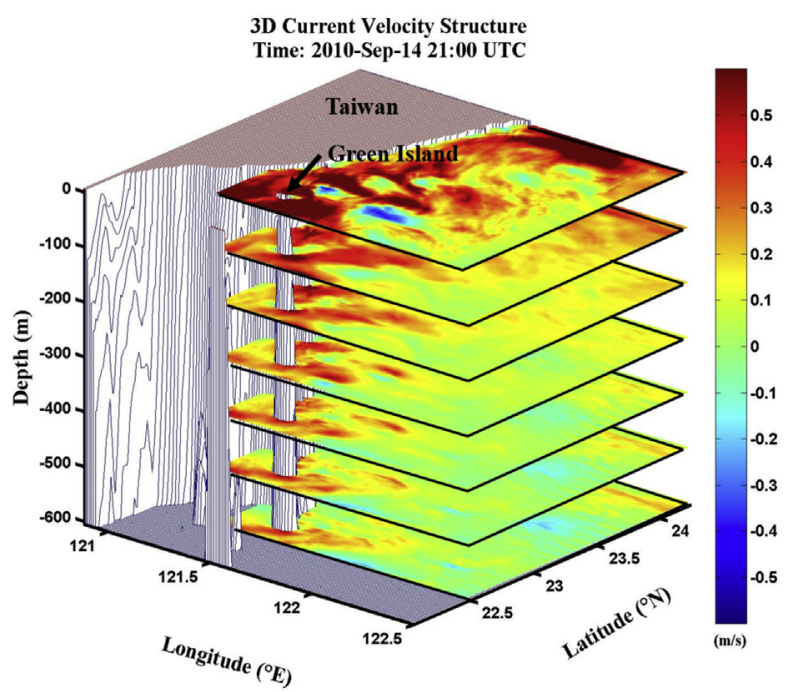

Fig. 12. Three-dimensional structure of $U$ velocity contours.
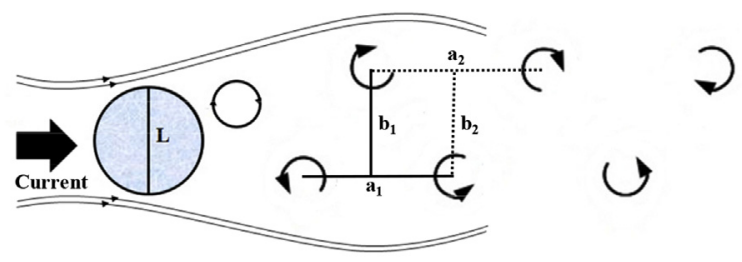

(a)

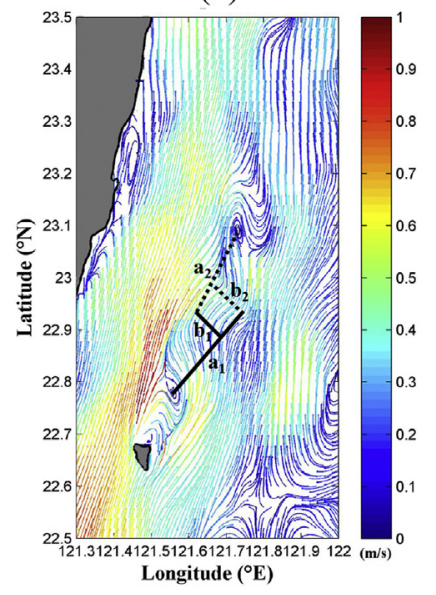

(b)

Fig. 13. (a) Schematic diagram for several definitions of the Von Kármán vortex street and (b) streamline of vector field at 08:00 UCT on 09/15/2010.

Table 4. Characteristics of Green Island vortex streets.

\begin{tabular}{lllll}
\hline Event & $\mathrm{a}$ & $\mathrm{b}$ & $\mathrm{Ar}$ & $\mathrm{Br}$ \\
\hline Present study & 25.97 & 7.32 & 3.64 & 1.46 \\
[18] & 24.19 & 12.51 & 1.98 & 2.50 \\
Case I in [21] & 17.9 & 10.2 & 1.76 & 1.92 \\
Case II in [21] & 27.7 & 10.2 & 2.72 & 1.84 \\
\hline
\end{tabular}


Table 5. Characteristics of Green Island wake at different tidal phases at a depth of $10 \mathrm{~m}$.

\begin{tabular}{llllll}
\hline Tide level & \multicolumn{2}{l}{ Model $[\mathrm{C}+\mathrm{T}+\mathrm{W}]$} & & \multicolumn{2}{l}{ Model $[\mathrm{C}+\mathrm{T}]$} \\
\cline { 2 - 3 } & High tide & Low tide & & High tide & Low tide \\
\hline $\mathrm{a}(\mathrm{km})$ & 31.0 & 27.1 & & 28.7 & 40.1 \\
$\mathrm{~b}(\mathrm{~km})$ & 4.9 & 8.1 & & 7.6 & 6.6 \\
$A r=a / b$ & 6.4 & 3.3 & & 3.8 & 6.1 \\
$\mathrm{Br}=b / L$ & 1.0 & 1.6 & & 1.5 & 1.3 \\
\hline
\end{tabular}

$U_{p}=U_{e} / u_{\infty}=a /\left(u_{\infty} T\right)$,

where $u_{\infty}$ and $T$ are the characteristic flow velocity and the period of the vortex shedding at a certain water depth, respectively. In addition, Ue is the eddy propagation speed.

Then, the dimensionless quantities $A r, B r, S t$, and $U p$ will be used to characterize the vortex shedding. The spatial lengths of $A r$ and $B r$ of the present model
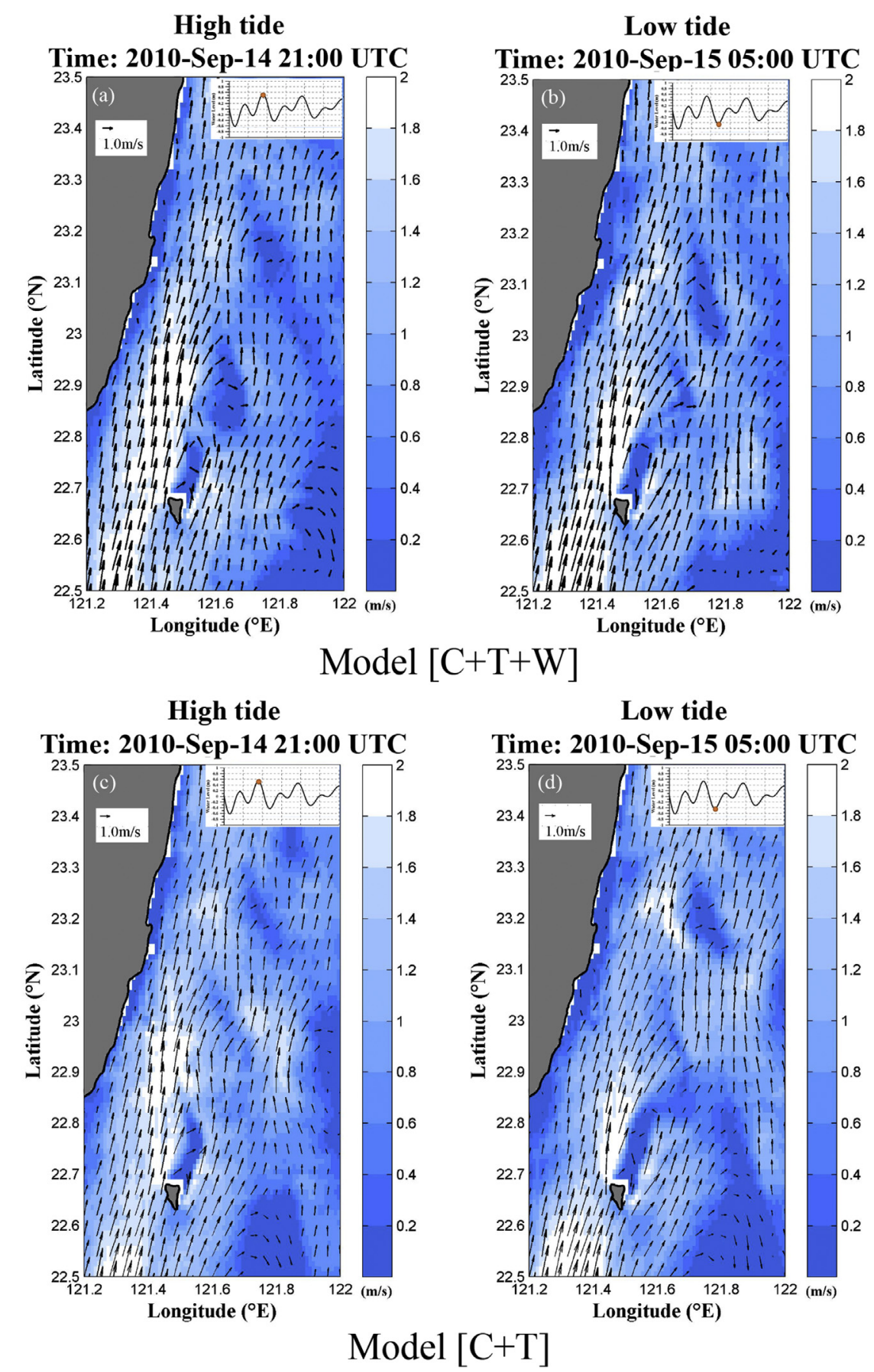

Fig. 14. Velocity fields corresponding to Table 5. 
Table 6. Comparison of computed velocity field with wind effect at different water depths at 9 pm on September 14, 2010.

\begin{tabular}{lllllll}
\hline Water depth $(\mathrm{m})$ & $\mathrm{a}$ & $\mathrm{b}$ & $\mathrm{Ar}$ & $\mathrm{Br}$ & $\mathrm{St}$ & $\mathrm{Up}$ \\
\hline 0 & 31.0 & 4.9 & 6.3 & 1.0 & 0.09 & 0.54 \\
50 & 27.4 & 7.0 & 3.9 & 1.4 & 0.29 & 1.58 \\
75 & 25.6 & 7.7 & 3.3 & 1.5 & 0.36 & 1.84 \\
100 & 23.1 & 13.1 & 1.8 & 2.6 & 0.36 & 1.18 \\
\hline
\end{tabular}

can be validated through a comparison with satellite image observations $[18,21]$. In Table 4 , the averaged values (A-G in Fig. $4 \mathrm{~b}$ ) of the present model are compared with the averaged data of 52 satellite images from 2002 to 2015 [18] and Cases I (July 30, 2003) \& Case II (March 29, 1999) in the other study [21]. Overall, the results are in good agreement with the satellite image observations except for a slight underestimation of the transverse length $(b)$ in the present model.

The spatial lengths are also evaluated for the high (21:00 UTC on 09/14/2010) and low (05:00 UTC on $09 / 15 / 2010$ ) tides by simulations with and without wind inputs. The results are shown in Table 5. In the Table, it can be observed that a larger aspect ratio is resulted during high tide (in the columns of Model $[C+\mathrm{T}+\mathrm{W}])$ as the Ekman effect of the SW monsoon and the high tide tend to elongate the vortex street. This elongation is also obvious if the two columns of high tides are compared. On the other hand, the effect is opposite for the cases of low tides. The corresponding velocity fields of the four cases are depicted in Fig. 14. The elongation can be observed in these figures.

Finally, the dimensional quantities $A r, B r, S t$, and $U p$ are evaluated for various water depths at $9 \mathrm{pm}$ on September 14, 2010. The results are summarized in Table 6. As this table evidently indicates, the vortex street becomes shorter and wider in deeper waters. In addition, the temporal scale of Strouhal number indicates that the vortex shedding is of higher frequency at the deeper waters. Finally, the relative propagation speed is higher at the mainstream depths of the Kuroshio currents.

\section{Conclusions}

In this paper, the three-dimensional Princeton Ocean Model was used to realistically simulate the Kuroshio-induced vortex shedding in the lee of Green Island. The numerical results were validated through comparisons with in-situ measurements from Green Island tidal station, the shipboard ADCP, CTD profiler, and MODIS satellite image datasets. Good agreements were presented for both two- and three-dimensional results. Because most of the previous numerical studies focus on two- dimensional velocity fields, the three-dimensional velocity, temperature, and salinity fields of the present model are compared well with the in-situ measurements from the shipboard A DCP and CTD profiler.

A scenario study was conducted to investigate the influence of the depth of the wind effect. The results indicate that the wind can affect the flow fields up to a depth of approximately $100 \mathrm{~m}$. Furthermore, the influence depth of the vortex shedding is shown to be deeper than $300 \mathrm{~m}$. Finally, an analysis was conducted to study the characteristics of vortex shedding, including the spatial lengths, temporal scales and propagation speeds. The results indicate that the Kuroshio-induced vortex street is elongated by the combined effects of the SW monsoon and the high tide. In addition, the vortex street became shorter, wider, and of higher frequency in the deeper waters. Further comparisons with the results in a recent study [20] will be investigated in the future.

\section{Acknowledgements}

This study was financially supported by the Ministry of Science and Technology (Taiwan), under the grant no. MOST 109-2221-E-992-046-MY3.

\section{References}

[1] Su Y-W. Electricity demand in industrial and service sectors in taiwan. Energy Efficiency 2018;11:1541-57.

[2] Chang Y-C, Chu PC, Tseng R-S. Site selection of ocean current power generation from drifter measurements. Renew Energy 2015;80:737-45.

[3] Chen F. The kuroshio power plant. London: Springer; 2013.

[4] Hsu T-W, Liau J-M, Liang S-J, Tzang S-Y, Doong D-J. Assessment of kuroshio current power test site of green island, taiwan. Renew Energy 2015c;81:853-63.

[5] Chao S-Y. Circulation of the east China sea, a numerical study. J Oceanogr 1990;46:273-95.

[6] Hsin YC, Wu CR, Shaw PT. Spatial and temporal variations of the kuroshio east of taiwan, 1982-2005: A numerical study. J Geophys Res: Oceans 2008;113.

[7] Su J, Guan B, Jiang J. The kuroshio. Part i. Physical. Oceanogr Marine Biol 1990;28:11-71.

[8] Jan S, Yang YJ, Wang J, Mensah V, Kuo TH, Chiou MD, et al. Large variability of the kuroshio at $23.75 \mathrm{n}$ east of taiwan. J Geophys Res: Oceans 2015;120:1825-40.

[9] Chen F. Kuroshio power plant development plan. Renew Sustain Energy Rev 2010;14:2655-68.

[10] Tang T, He C, Wang Y, Jan S, Hsu S, Wen L, et al. Comprehensive research on the natural resources of the east waters of taiwan: A precisely topographic, geological, hydrological, and ecological surveys of the waters around green island. A research report of the project sponsored by. National Science Council; 2008.

[11] Von Kármán T. Aerodynamics. New York: McGraw-Hill; 1954.

[12] Hsu T-W, Chou M-H, Hou T-H, Liang S-J. Typhoon effect on kuroshio and green island wake: A modelling study. Ocean Sci Discuss 2015a;12:3199-233.

[13] Hsu T-W, Doong D-J, Hsieh K-J, Liang S-J. Numerical study of monsoon effect on green island wake. J Coastal Res 2015b; 31:1141-50. 
[14] Jiménez B, Sangra P, Mason E. A numerical study of the relative importance of wind and topographic forcing on oceanic eddy shedding by tall, deep water islands. Ocean Model 2008;22:146-57.

[15] Chang MH, Tang TY, Ho CR, Chao SY. Kuroshio-induced wake in the lee of green island off taiwan. J Geophys Res: Oceans 2013;118:1508-19.

[16] Chang M-H, Jan S, Liu C-L, Cheng Y-H, Mensah V. Observations of island wakes at high rossby numbers: Evolution of submesoscale vortices and free shear layers. J Phys Oceanogr 2019;49:2997-3016.

[17] Chang M-H, Jan S, Mensah V, Andres M, Rainville L, Yang $\mathrm{YJ}$, et al. Zonal migration and transport variations of the kuroshio east of taiwan induced by eddy impingements. Deep Sea Res I: Oceanogr Res Papers 2018;131:1-15.

[18] Hsu P-C, Chang M-H, Lin C-C, Huang S-J, Ho C-R. Investigation of the island-induced ocean vortex train of the kuroshio current using satellite imagery. Rem Sens Environ 2017;193:54-64.

[19] Hsu P-C, Cheng K-H, Jan S, Lee H-J, Ho C-R. Vertical structure and surface patterns of green island wakes induced by the kuroshio. Deep Sea Res Part I: Oceanogr Res Papers 2019;143:1-16.

[20] Hsu P-C, Ho C-Y, Lee H-J, Lu C-Y, Ho C-R. Temporal variation and spatial structure of the kuroshio-induced submesoscale island vortices observed from gcom-c and himawari-8 data. Rem Sens 2020;12:883.

[21] Huang S-J, Ho C-R, Lin S-L, Liang S-J. Spatial-temporal scales of green island wake due to passing of the kuroshio current. Int J Rem Sens 2014;35:4484-95.

[22] Liang S-J, Lin C-Y, Hsu T-W, Ho C-R, Chang M-H. Numerical study of vortex characteristics near green island, taiwan. J Coast Res 2013;29:1436-44.

[23] Liu CL, Chang MH. Numerical studies of submesoscale island wakes in the kuroshio. J Geophys Res: Oceans 2018;123: 5669-87.

[24] Mellor GL, Yamada T. A hierarchy of turbulence closure models for planetary boundary layers. J Atmos Sci 1974;31: 1791-806.
[25] Mellor GL, Yamada T. Development of a turbulence closure model for geophysical fluid problems. Rev Geophys 1982;20: 851-75.

[26] Flather R. A tidal model of the northwest european continental shelf. Mem Soc Roy Sci Liege 1976;10:141-64.

[27] Blumberg AF, Mellor GL. A description of a three-dimensional coastal ocean circulation model. Three-Dimension Coast Ocean Models 1987;4:1-16.

[28] Amante C, Eakins BW. Etopo1 1 arc-minute global relief model: Procedures, data sources and analysis. US Department of Commerce, National Oceanic and Atmospheric Administration, National Environmental Satellite, Data, and Information Service, National Geophysical Data Center, Marine Geology and Geophysics Division Colorado; 2009.

[29] MOST. In: Ocean data bank of the ministry of science and technology; 2011. http://www.odb.ntu.edu.tw/ Aaa.

[30] Juang $\mathrm{H}-\mathrm{MH}$, Kanamitsu $\mathrm{M}$. The nmc nested regional spectral model. Monthly Weather Rev 1994;122:3-26.

[31] Egbert GD, Bennett AF, Foreman MG. Topex/poseidon tides estimated using a global inverse model. J Geophy Res: Oceans 1994;99:24821-52.

[32] Egbert GD, Erofeeva SY. Efficient inverse modeling of barotropic ocean tides. J Atmos Oceanic Technol 2002;19: 183-204.

[33] Chassignet EP, Hurlburt HE, Smedstad OM, Halliwell GR, Hogan PJ, Wallcraft AJ, et al. The hycom (hybrid coordinate ocean model) data assimilative system. J Marine Syst 2007;65: 60-83.

[34] Mellor GL. Users guide for a three dimensional, primitive equation, numerical ocean model. In: Program in atmospheric and oceanic sciences. Princeton, NJ: Princeton University; 1998. 08544-0710.

[35] Anthes RA. Data assimilation and initialization of hurricane prediction models. J Atmos Sci 1974;31:702-19.

[36] Hoke JE, Anthes RA. The initialization of numerical models by a dynamic-initialization technique. Monthly Weather Rev 1976;104:1551-6. 